\title{
The Relation between Spin and Orbital Periods in HMXBs
}

\author{
Q. Z. $\mathrm{Liu}^{1}$, X. D. $\mathrm{Li}^{2}$, and D. M. Wei ${ }^{1}$ \\ ${ }^{1}$ Purple Mountain Observatory, Academia Sinica, Nanjing 210008, \\ China \\ ${ }^{2}$ Department of Astronomy, Nanjing University, Nanjing 210093, China
}

\begin{abstract}
The relation between the spin period $\left(P_{s}\right)$ and the orbital period $\left(P_{o}\right)$ in high-mass X-ray binaries (HMXBs) is investigated. In order for Be/X-ray binaries to locate above the critical line of observable $\mathrm{X}$-ray emission due to accretion, it is necessary for an intermediate orbital eccentricity to be introduced. We suggest that some peculiar systems in the $P_{o}-P_{s}$ diagram are caused by their peculiar magnetic fields.
\end{abstract}

\section{Introduction}

HMXBs consist of a neutron star or a black hole, orbiting an early-type massive star. They can be further divided into Be/X-ray binaries and SG/X-ray binaries. Corbet (1986) showed that HMXBs fall into three separate groups when the pulse period is plotted versus the orbital period. Those systems with Be companions have a strong correlation between the orbital and spin periods, while systems with wind-fed and disk-fed OB supergiant companions fall into two separate broad regions of the diagram. This correlation can be interpreted in terms of the equilibrium periods of accreting magnetized neutron stars.

\section{The relation between spin and orbital periods}

So far about 40 systems both reliable orbital and spin periods are known (Liu et al. 2000). The spin period and the orbital period of all probable massive X-ray binaries for which both values are known are plotted in Fig. 1. The Be-systems, the wind-fed systems, and the disk-fed systems are indicated by filled circles, open circles and crosses, respectively. The figure shows that these three types of systems occupy different parts of the diagram, confirmed the conclusion of Corbet (1986) although with some scatter. Also included in the figure are the Be-radio pulsar systems (squares). According to Waters \& van Kerkwijk (1989), a $P_{o}-P_{s}$ relation for observable Be/X-ray binaries can be obtained

$$
P_{s}=2^{\frac{3}{2}} \frac{\pi^{4 / 7}\left(B R_{n}^{3}\right)^{6 / 7}}{\left(2 G M_{n}\right)^{11 / 7}}\left(\frac{\rho_{0}}{v_{r 0}^{3}}\right)^{-3 / 7}\left(\frac{v_{r}}{v_{r e l}}\right)^{-9 / 7}\left[\frac{G\left(M_{n}+M_{*}\right)(1-e)^{3}}{4 \pi^{2} R_{*}^{3}} P_{o}^{2}\right]^{(4 n-6) / 7} .
$$

All parameters refer to their usual meaning. With typical values of the parameters, we can plot in Fig. 1 the critical lines (solid lines) of observable X-ray emission for different eccentricities. It is clear that an eccentricity of about 
0.3 should be introduced in order for all Be/X-ray binaries to locate above the critical line, while $\mathrm{Be} / \mathrm{RP}$ systems are below the line due to the centrifugal mechanism.

If the mass-donor star is a supergiant, or a Be star with only pure stellar wind, the dense and slow equatorial wind would be replaced by a much weaker and faster polar wind. The equilibrium period will be obtained at

$$
P_{s}=2^{33 / 14} \frac{\pi\left(a B R_{n}^{3}\right)^{6 / 7}}{\left(2 G M_{n}\right)^{11 / 7}}\left(\frac{v_{r}}{v_{r e l}}\right)^{-9 / 7} \dot{M}_{w}^{-3 / 7} v_{w}(a)^{12 / 7}
$$

The dash lines are the theoretical lines of wind-fed X-ray binaries for a neutron star spinning at its equilibrium period with different magnetic fields.

\section{Peculiar systems and their implications}

We can see from Fig. 1 that two SG/X-ray binaries, OAO 1657-415 and $2 \mathrm{~S}$ $0114+650$, fall high above and below the wind-fed SG/X-ray binary region, respectively. The Be/X-ray binaries, AX J0051-733 and A0538-66, are also well off the $\mathrm{Be} / \mathrm{X}$-ray binary region. $2 \mathrm{~S} 0114+650$ was probably born as a magnetar (Li \& van den Heuvel 1999), and the magnetic field in A0538-66 is likely weak. According to Eqs. (1) and (2), we can calculate the magnetic field of the neutron stars in OAO 1657-415 and AX J0051-733. We find that the magnetic field in OAO $1657-415$ is about $2 \times 10^{11}-6.7 \times 10^{11} \mathrm{G}$, one order of magnitude lower than the typical magnetic field in HMXBs. We suggest that the pulsar in AX J0051-733 is likely born with ultrostrong magnetic field strength, between $2.5 \times 10^{13}-5 \times 10^{15} \mathrm{G}$, thus probably the descendant of a magnetar.

Acknowledgments. This work is supported by the 973 Project through Grant G1999075405 and by NSFC through Grants 10173026 and 10073022.

\section{References}

Corbet, R. H. D. 1986, MNRAS, 220, 1047

Li, X. D. \& van den Heuvel, E. P. J. 1999, ApJ, 513, L45

Liu, Q. Z., van Paradijs, J., \& van den Heuvel, E. P. J. 2000, A\&AS, 147, 25

Waters, L. B. F. M. \& van Kerkwijk, M. H. 1989, A\&A, 223, 196 


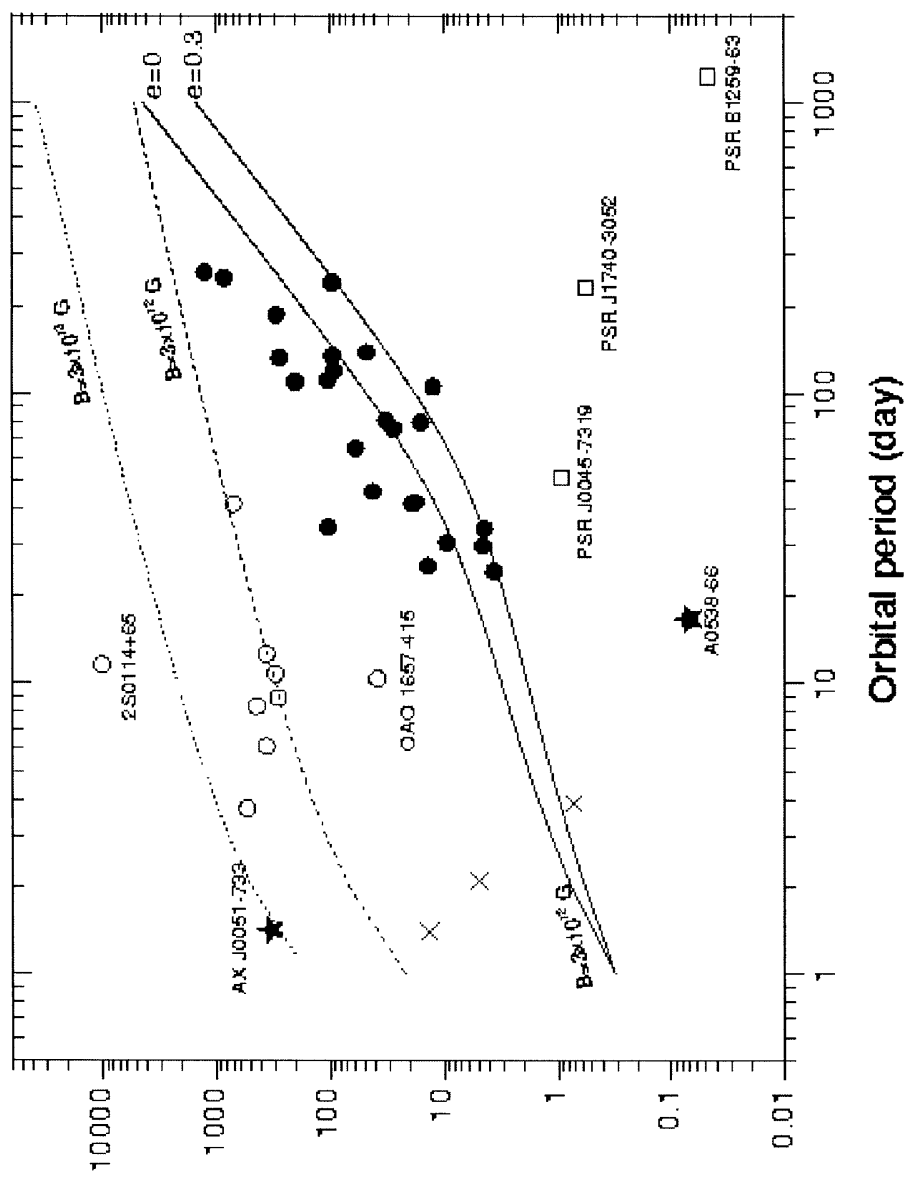

(s) polpod es|nd

Figure 1. The relation between orbital and spin periods. 